The Astrophysical Journal, 671:1868-1876, 2007 December 20

(C) 2007. The American Astronomical Society. All rights reserved. Printed in U.S.A.

\title{
AN ESTIMATION OF THE GAMMA-RAY BURST AFTERGLOW APPARENT OPTICAL BRIGHTNESS DISTRIBUTION FUNCTION
}

\author{
Carl W. Akerlof ${ }^{1}$ and Heather F. Swan ${ }^{1}$ \\ Received 2006 September 29; accepted 2007 September 6
}

\begin{abstract}
By using recent publicly available observational data obtained in conjunction with the NASA Swift gamma-ray burst (GRB) mission and a novel data analysis technique, we have been able to make some rough estimates of the GRB afterglow apparent optical brightness distribution function. The results suggest that $71 \%$ of all burst afterglows have optical magnitudes with $m_{R}<22.1$ at $1000 \mathrm{~s}$ after the burst onset, the dimmest detected object in the data sample. There is a strong indication that the apparent optical magnitude distribution function peaks at $m_{R} \approx 19.5$. Such estimates may prove useful in guiding future plans to improve GRB counterpart observation programs. The employed numerical techniques might find application in a variety of other data analysis problems in which the intrinsic distributions must be inferred from a heterogeneous sample.
\end{abstract}

Subject headings: gamma rays: bursts — methods: statistical

\section{INTRODUCTION}

One of the outstanding questions about gamma-ray burst (GRB) afterglows is their optical luminosity. Since the first counterpart (van Paradijs et al. 1997) was identified in 1997 February 28, GRBs have been detected optically over an intensity range that spans at least 14 mag using instruments ranging in aperture from $10 \mathrm{~cm}$ to $10 \mathrm{~m}$. Although the NASA Swift mission successfully determines celestial coordinates to accuracies that are often better than a few arcseconds, less than $50 \%$ of all Swift detections have led to identifiable optical counterparts. The reason for this relatively low rate has been the subject of much speculation. The three popular views are that: (1) GRBs are born in dusty, opaque star-forming regions (Reichart \& Price 2002; Klose et al. 2003; Vergani et al. 2004; Levan et al. 2006); or (2) originate at redshifts that make them invisible to us at optical wavelengths (Jakobsson et al. 2004, 2006); or (3) are intrinsically dimmer than average (Fynbo et al. 2001; Rol et al. 2005). No doubt, the truth is some combination of these possibilities. We do not address these questions directly in this paper. Instead, we have tried to estimate the fraction of bursts with afterglows that are reasonably accessible to detection with observatories now in existence. We have developed a fairly simple procedure for using the reported instrumental detection thresholds in conjunction with the actual distribution of detected magnitudes to infer the underlying apparent afterglow optical brightness function.

\section{OVERVIEW OF ANALYSIS TECHNIQUE}

There are now more than 200 Swift GRB detections since the launch of this mission on 2004 November 20. The world community of ground-based astronomers has responded with optical observations of essentially all of these events, greatly augmenting the onboard measurements of the Swift UVOT camera. From the data that have been reported, principally via the GCN, one can obtain the optical brightness for detected events, $m_{\mathrm{det}}$, and the limiting magnitudes, $m_{\text {lim }}$, for those that are not. With this primary data, we have estimated the detected and limiting magnitudes at a fixed time of $1000 \mathrm{~s}$ postburst and extrapolated the limiting magnitude data to include the detected events as well. For each of these steps, we will demonstrate that the statistical

\footnotetext{
${ }^{1}$ University of Michigan, Randall Laboratory of Physics, 450 Church Street, Ann Arbor, MI 48109-1040; cakerlof@umich.edu, hswan@umich.edu.
}

techniques appear to be quite robust. This is principally due to the fact that the analysis is based solely on cumulative probability distributions for $m_{\mathrm{det}}$ and $m_{\mathrm{lim}}$. Thus, estimation errors for individual events tend to get washed out in the mean as long as gross systematic effects are avoided. It is easy to see that one reason that less than $50 \%$ of all bursts have detected optical counterparts is due to the limited sensitivities of the ensemble of instruments that was available at any given time. That can be framed more precisely by assuming that nature has provided some intrinsic optical afterglow luminosity distribution to us on Earth, specified in magnitudes. For each GRB detected by Swift, there is one best observational limiting magnitude. The convolution of these two distributions must be the observed distribution of $m_{\text {det }}$. This equality can be converted to an optimization problem of finding the best intrinsic afterglow distribution that satisfies this constraint. This estimate is probably the best we can do with the extremely heterogeneous observations that have been reported and the finite statistics of the sample.

\section{DATA SELECTION AND CORRECTION}

The data for the ensuing analysis were collected from 118 Swift-identified GRBs that spanned a 447 day period from 2005 February 15 through 2006 May 7. Both the GRB detection magnitudes and limiting magnitudes were subjected to some identical selection criteria and corrections. Foremost, the midpoint of the optical observations were required to lie within a factor of 10 of a nominal postburst time of 1000 s, i.e., between 100 and $10,000 \mathrm{~s}$. Observations were restricted to $V$ or $R$ band with unfiltered counting as $R$. These restrictions eliminated 10 bursts from further consideration: nine due to the time cut and one due to the observing wavelength ( $K_{s}$ band). Explicitly, a few events were labeled as nondetections when the only actual detections evaded the allowed time window or filter constraints. All magnitudes were also compensated for galactic absorption using the NASA/ IPAC Extragalactic Database Web-based calculator ${ }^{2}$ that, in turn, is based on the work of Schlegel et al. (1998). For data taken under $V$ filters, a further adjustment of $-0.41 \mathrm{mag}$ was applied to compensate for the average GRB color difference between $V$ and $R$. This adjustment is the average difference between $V$ and $R$ for time periods ranging from 0.2 to 1 days for five GRBs that had

\footnotetext{
${ }^{2}$ See http://nedwww.ipac.caltech.edu/forms/calculator.html.
} 
many measurements of $V$ and $R$ at many different times: 990510 (Stanek et al. 1999), 021004 (Bersier et al. 2003), 050502A (Guidorzi et al. 2005b), 020813 (Covino et al. 2003), and 030329 (Burenin et al. 2003; Rumyantsev et al. 2003; Zharikov et al. 2003). The light curves were characterized by identical powerlaw decays, so there is no evidence of chromatic variability over these time spans.

Beyond this point, the additional selection criteria for $m_{\text {det }}$ and $m_{\text {lim }}$ somewhat diverged. For each of the 43 events with valid detections, the measurement with an observation time logarithmically closest to $1000 \mathrm{~s}$ was chosen. The list is displayed in Table 1. (Much of the data for this paper were obtained from the GRBlog Web pages $^{3}$ maintained by Quimby et al. [2003], which enormously facilitated this project.) In order to proceed further, we must compare the optical brightnesses at a common postburst time delay.

To make this project work, we needed to establish that it was possible to extrapolate each observed magnitude at $t$ in the range $[100,10,000]$ to a fixed time, $t_{c}=1000 \mathrm{~s}$. Fortunately, there were sufficient data for 37 of the 43 events to extract a power-law exponent, $\alpha$, for the temporal behavior of each burst. With these values, we could make a reasonable estimate of $m_{\text {det }}$ at $t_{c}$. We also performed a similar calculation assuming a fixed value for $\alpha=-0.70$. The two cumulative probability distributions for the extrapolated values of $m_{\text {det }}$ are plotted in Figure 1. Application of the Smirnov-Cramér-von Mises test shows that the two distributions are effectively identical (Eadie et al. 1971, p. 268; Kendall \& Stuart 1979, p. 474). This gives us some confidence that the same power-law extrapolation is appropriate when the burst afterglows are NOT detected. This is verified by looking at the cumulative distributions of the observation times for the detections and nondetection upper limits (to be described below). This is shown in Figure 2. As expected, the detected events lie close to $t_{c}$ by virtue of the imposed selection criteria. The undetected events have no such bias. Nevertheless, their median lies close to $1000 \mathrm{~s}$ as well. We can make this more quantitative by comparing the rms average magnitude shifts for the detections and upper limits due to translating from $t_{\text {burst }}$ to $t_{c}$. With $\alpha=-0.70$, the average detected magnitude is shifted by 0.59 when extrapolating from $t_{\text {burst }}$ to $t_{c}$, while the similar number for upper limits is 1.06 . Thus, the estimated cumulative distribution for the upper limits will be somewhat poorer, but the plots in Figure 1 demonstrate that this is unlikely to be significant.

The estimation of the instrumental upper limits for afterglows, $m_{\text {lim }}$, is more complex. First of all, very few research groups report $m_{\lim }$ if there has been a detection. Even if they do, there is a serious bias that will tend to shift $m_{\mathrm{lim}}$ to greater values: a large telescope is much more likely to observe a GRB if the optical counterpart has already been announced. We have found a slightly devious way to get around these difficulties by using the unbiased limiting magnitude distribution for nondetections to estimate the limiting magnitude distribution for all bursts. For each undetected GRB, all limiting magnitude reports are transformed as if they were detections to $t_{c}$, only requiring an observation time within the $[100,10,000] \mathrm{s}$ window. The maximum magnitude of each set is adopted as $m_{\text {lim }}$ for that burst; 65 events survived this analysis and are listed in Table 2.

Our task now is to create a distribution of all limiting magnitudes, both detected and undetected, knowing only the values for the undetected. One obvious fact is that the limiting magnitudes for detections will, on average, be deeper. In fact, if a de-

\footnotetext{
3 See http://grad40.as.utexas.edu/.
}

tection is made at $m_{\text {det }}$, the value for $m_{\text {lim }}$ will lie somewhere between $m_{\text {det }}+\sigma_{\text {det }}$ (where $\sigma_{\text {det }}$ is the measurement error associated with $m_{\text {det }}$ ) and the best limiting magnitude ever reported. If $m_{\text {lim }}$ truly represents the maximum sensitivities for the ensemble of bursts, the simplest tactic is to take the median of the subset of $m_{\text {lim }}$ in the prescribed range and incorporate that value into the entire set of $m_{\mathrm{lim}}$. By performing this recursively over the set of detected GRB afterglows, ordered by decreasing $m_{\text {det }}$, one can fill out the otherwise missing entries.

We carried this one step further to better understand the stability of this method. We generated $1001 m_{\text {lim }}$ distributions using a uniform random number generator to select the interpolated values. For each successive element of $m_{\text {det }}$, a modified subset of $m_{\text {lim }}$ is considered that includes all elements of $m_{\text {lim }}$ with values greater than $m_{\text {det }}+\sigma_{\text {det }}$ adjoined to the lower limit value. A uniformly distributed random number then uses the cumulative distribution of the restricted set to select an appropriate random value to be adjoined to $m_{\mathrm{lim}}$. In the limit of a large sample of $m_{\text {lim }}$ distributions, all possible sets for $m_{\lim }$ will be generated consistent with the constraints imposed by the values for the undetected $m_{\text {lim }}$ and the detected $m_{\text {det }}$. To recover the best estimate for $m_{\text {lim }}$, the 1001 distributions were individually ordered by value. To select the 108 elements of $m_{\text {lim }}$, the first value was chosen as the median of the set of first values of the 1001 Monte Carlo sets, the second value from the set of second values, etc. A similar procedure defines the first and third quartile distributions. If the distribution of such sets is tightly confined, we have reason to anticipate that this is an adequate approximation of reality. The results are shown in Figure 3. The median distribution lies within tight bounds constrained by the first and third quartiles.

The validity of this procedure was verified by modeling this deconvolution process assuming knowledge of the true $m_{\mathrm{lim}}$ distribution. For the sake of computational simplicity, the $m_{\text {lim }}$ cumulative distribution was approximated by a Fermi-Dirac distribution with the two free parameters chosen to best fit the apparent shape inferred from the analysis described above. The $m_{\text {det }}$ distribution was taken from the four-parameter B-spline representation described in $\S 4$ below. This allowed us to create for $N$ events, a list of simulated Monte Carlo GRBs with values for the afterglow and limiting instrumental detector magnitudes determined by the two assumed cumulative distributions. Comparing the two values, event by event, generated two subsamples: the "detected" events for which the afterglow was brighter than the instrumental limit and the "undetected" events for which the opposite was true. The Monte Carlo samples reproduced the detected/undetected event ratios essentially exactly. Applying the deconvolution scheme that has been described, we found excellent agreement with the input assumptions for the distribution of $m_{\mathrm{lim}}$. One reason for the stability of this technique is the broad dispersion of sensitivities of groundbased instruments reporting results. One measure is the distribution of apertures: it is approximately logarithmic from 0.2 to $8.2 \mathrm{~m}$ with $d N \propto d$ (aperture)/aperture.

Figure 4 shows the histogram distributions of detected GRBs and the limiting magnitudes for nondetections, both scaled to $t_{c}=$ $1000 \mathrm{~s}$. The distributions are roughly similar with the latter edging just a bit deeper. Such rough equality is what one might naively expect for the situation in which about half of all events evade detection. Above $m_{R}=19$, there are twice as many nondetections (29) as detections (14).

\section{FINDING THE OPTICAL BRIGHTNESS DISTRIBUTION FUNCTION}

The basic idea of this calculation is to specify the apparent optical brightness function by a small set of parameters and, with 
TABLE 1

GRB Afterglow Detections

\begin{tabular}{|c|c|c|c|c|c|c|c|c|c|c|}
\hline 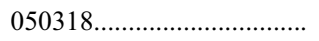 & 031851.15 & -462343.70 & V & 0.054 & 0.043 & -0.87 & 17.80 & 3230.00 & 16.445 & 1 \\
\hline $050401 \ldots \ldots \ldots \ldots$ & 163128.82 & +021114.83 & None & 0.216 & 0.174 & -0.76 & 18.58 & 241.35 & 19.486 & 3 \\
\hline 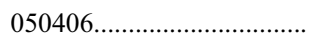 & 021752.30 & $-5011 \quad 15.00$ & V & 0.073 & 0.059 & -0.75 & 19.44 & 138.00 & 20.462 & 4 \\
\hline 050416А .............................. & 123354.60 & +210324.00 & V & 0.098 & 0.079 & * & 19.38 & 115.00 & 20.516 & 5 \\
\hline 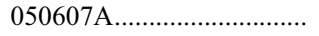 & 200042.79 & +090831.50 & $R$ & 0.516 & 0.416 & -1.00 & 22.50 & 960.00 & 22.115 & 8 \\
\hline 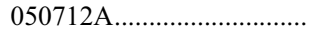 & 051047.90 & +645451.50 & $V$ & 0.753 & 0.607 & -0.73 & 17.38 & 959.00 & 16.249 & 9 \\
\hline 050713А & 212209.53 & +770429.50 & $R$ & 1.371 & 1.106 & -0.67 & 21.41 & 2963.00 & 19.478 & 10 \\
\hline 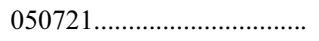 & 165344.53 & -282251.80 & $R$ & 0.894 & 0.721 & -1.29 & 17.93 & 1484.00 & 16.909 & 11 \\
\hline $050726 \ldots \ldots \ldots \ldots$ & 132012.30 & -320350.80 & $V$ & 0.206 & 0.166 & 0 & 17.35 & 173.00 & 18.067 & 12 \\
\hline 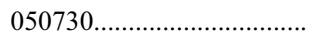 & 140817.13 & -034616.70 & $R$ & 0.168 & 0.135 & -0.54 & 17.07 & 1848.00 & 16.468 & 13 \\
\hline 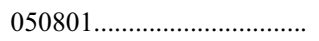 & 133635.00 & -215541.00 & None & 0.319 & 0.257 & -1.31 & 16.93 & 996.00 & 16.676 & 14 \\
\hline 050908 & 012150.75 & -125717.20 & $R c$ & 0.083 & 0.067 & -0.93 & 18.80 & 900.00 & 18.813 & 19 \\
\hline 050922C & 210933.30 & -084527.50 & None & 0.342 & 0.276 & -1.00 & 16.00 & 640.00 & 16.063 & 20 \\
\hline 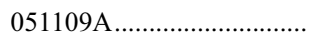 & 220115.31 & +404923.31 & None & 0.630 & 0.508 & -0.65 & 17.59 & 1004.00 & 17.079 & 21 \\
\hline $051111 \ldots \ldots \ldots$ & 231233.36 & +182229.53 & None & 0.537 & 0.433 & -0.74 & 16.13 & 1007.00 & 15.692 & 21 \\
\hline 051117A ............................ & 151334.09 & +305212.70 & V & 0.080 & 0.065 & -0.35 & 20.01 & 210.00 & 20.706 & 22 \\
\hline 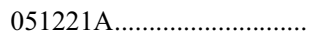 & 215448.63 & +165327.16 & $R$ & 0.227 & 0.183 & -0.93 & 20.20 & 4680.00 & 18.844 & 23 \\
\hline 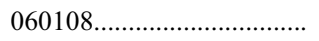 & 094801.98 & +315508.60 & $R$ & 0.059 & 0.047 & -0.43 & 21.84 & 879.00 & 21.891 & 24 \\
\hline 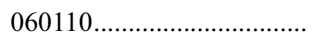 & 045057.85 & +282555.70 & None & 2.107 & 1.699 & -0.70 & 17.90 & 847.00 & 16.327 & 25 \\
\hline 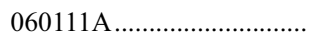 & 182449.00 & +373616.10 & None & 0.094 & 0.076 & * & 18.30 & 173.50 & 19.555 & 26 \\
\hline 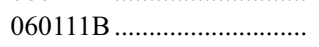 & 190542.47 & +702233.10 & None & 0.368 & 0.297 & -1.08 & 18.90 & 792.00 & 18.780 & 27 \\
\hline 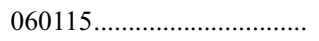 & 033608.40 & +172043.00 & $R c$ & 0.441 & 0.356 & 0.00 & 19.10 & 1190.00 & 18.612 & 28 \\
\hline 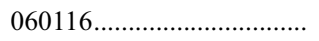 & 053846.28 & -052613.14 & None & 0.873 & 0.704 & -1.09 & 20.78 & 926.08 & 20.134 & 29 \\
\hline 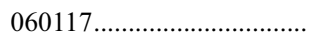 & 215136.13 & +595839.10 & $R$ & 4.292 & 0.010 & -1.70 & 12.62 & 502.90 & 13.132 & 30 \\
\hline 060323 & 113745.40 & +495905.50 & None & 0.050 & 0.040 & $*$ & 18.20 & 540.00 & 18.628 & 39 \\
\hline $060418 \ldots \ldots \ldots$ & 154542.40 & -033822.80 & $R c$ & 0.743 & 0.599 & -1.20 & 16.47 & 2412.00 & 15.202 & 40 \\
\hline 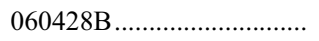 & 154125.63 & +620130.30 & None & 0.049 & 0.040 & 0.05 & 19.64 & 1013.00 & 19.590 & 41 \\
\hline 060502A & 160342.48 & +663602.50 & $R$ & 0.109 & 0.088 & -0.45 & 19.80 & 2400.00 & 19.047 & 42 \\
\hline
\end{tabular}

NotE.- Units of right ascension are hours, minutes, and seconds, and units of declination are degrees, arcminutes, and arcseconds.

${ }^{\text {a }}$ The quantity $m_{\text {det }}$ is the measured magnitude at $t_{\text {burst }}$ seconds after the GRB trigger.

$\mathrm{b}$ The quantity $m_{\mathrm{det}} @ t_{c}$ is the inferred value for $m_{\operatorname{det}}$ at $t_{c}=1000 \mathrm{~s}$ after correcting for galactic absorption and average GRB color differences.

References.-(1) Still et al. 2005; (2) Quimby et al. 2006b; (3) Rykoff et al. 2005c; (4) Schady et al. 2006; (5) Schady et al. 2005a; (6) Klotz et al. 2005a; (7) Klotz et al. 2005d; (8) Pagani et al. 2006; (9) De Pasquale et al. 2006a; (10) Guetta et al. 2007; (11) Antonelli et al. 2006; (12) Poole et al. 2005b; (13) Pandey et al. 2006; (14) Rykoff et al. 2006b; (15) McGowan et al. 2005a; (16) Schady et al. 2005b; (17) Cenko et al. 2006b; (18) Lipunov et al. 2005; (19) Torii 2005a; (20) Rykoff et al. 2005a; (21) Yost et al. 2007; (22) Holland et al. 2005a; (23) Wren et al. 2005; (24) Oates et al. 2006; (25) Li 2006; (26) Klotz et al. 2006a; (27) Klotz et al. 2006b; (28) Yanagisawa et al. 2006; (29) H. F. Swan et al. 2008, in preparation; (30) Jelínek et al. 2006; (31) Romano et al. 2006; (32) Bikmaev et al. 2006; (33) Guidorzi et al. 2006; (34) Monfardini et al. 2006a; (35) Stanek et al. 2007; (36) Quimby et al. 2006a; (37) Marshall et al. 2006; (38) Cobb 2006; (39) Zheng et al. 2006; (40) Koppelman 2006; (41) Li et al. 2006; (42) Cenko et al. 2006a.

this input, estimate the magnitude distribution of detected events modulated by the actual probability of making such a set of measurements with the required threshold sensitivity. By the usual least-squares techniques, the parameter set describing the afterglow brightness function is adjusted so that the predicted distribution of detections closely matches the actual measurements. With that in mind, we originally set out to represent the integral brightness distribution function, $F(m)$, by a set of cubic B-splines uniformly spaced over the range of observed magnitudes. Work- ing with the integral distribution function removes the ambiguity of selecting the binning interval that is implicitly required for defining the associated differential distribution. However, the tradeoff is that the representation of the integral distribution must guarantee that the function is monotonic over its entire range. In detail, it was realized that computing $F$ as a function of the magnitude, $m$, led to problems near the endpoints where $F$ must approach either zero or 1. Inverting the representation so that $m(F)$ is described by uniform $B$-splines over the interval, $[0,1]$, takes care of the 


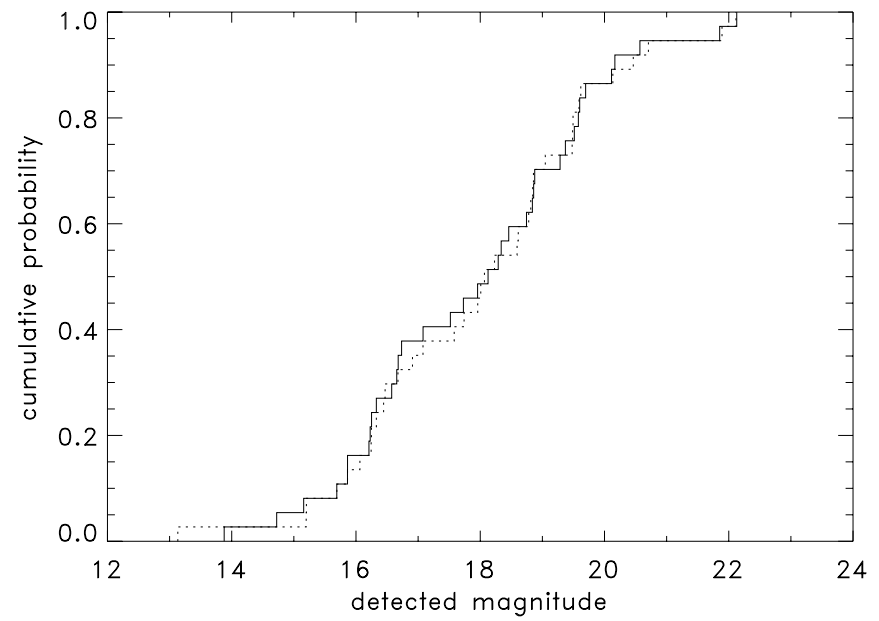

FIG. 1.-Cumulative distributions of afterglow magnitudes for 37 detected GRBs transformed to $t_{c}=1000 \mathrm{~s}$ according to a power-law extrapolation. The solid line shows the distribution using a value of $\alpha$ computed individually for each burst; the dotted line represents the similar distribution when $\alpha$ is set to a fixed value of -0.70 for all events. There is no apparent statistical difference between these curves.

endpoint problem nicely, although at the expense of denying solution by linear regression.

Despite some misgivings about poor computational speed, it was found that the downhill simplex minimization method of Nelder \& Mead (1965) was quite capable of finding solutions quickly for spline curves defined by up to 7 degrees of freedom. The IDL numerical analysis package ${ }^{4}$ was used for these computations, in particular the AMOEBA routine adapted from $\S 10.4$ of Numerical Recipes in C (2nd ed.; Press et al. 1992). This approach made it convenient to enforce the monotonicity of the integral distribution function - whenever an evaluation of the goodness-of-fit function was requested with B-spline coefficients leading to zeros or negative values of the distribution function derivative, $d m / d F$, the returned value was set to exceed the maximum of all previous values over the simplex. Thus, nonmonotonic integral distributions were easily rejected along with other computational problems.

As sketched above, we fold the estimated detection limiting magnitude distribution with a parametrically defined function describing the true GRB afterglow distribution to predict the observed distribution of actual detections. The starting point for this calculation is the integral distribution of detection upper limits, $m_{\text {lim }}$, described earlier. This is a staircase function with uniform vertical steps between irregular intervals, $\Delta m=m_{i}-$ $m_{i-1}$, in which the probability of observing with a given limiting sensitivity, $p_{i} \equiv p\left(m_{i-1} \rightarrow m_{i}\right)$, is uniform. Within each of these intervals of magnitude, the expected number of detected GRB events will increase by an amount, $\Delta f_{i}^{\text {calc }}=\Delta F_{i} p_{i}$, where $\Delta F_{i}=F\left(m_{i}\right)-F\left(m_{i-1}\right)$ is the associated change in the optical brightness distribution function over $\Delta m$. The sequence of values for $F\left(m_{i}\right)$ are computed by inversion of the cubic spline representation, $m(F)$.

Once the set of $\Delta f_{i}^{\text {calc }}$ is constructed, the cumulative probability distribution for the expected number of detected events can be obtained by summation: $f_{i}^{\text {calc }}=\sum^{i} \Delta f_{j}^{\text {calc }}$. A trivial modification of this procedure allows one to compute $f^{\text {calc }}$ for the sequence of ordered values of $m_{\text {det }}$ that characterize the actual GRB detections. The experimentally observed cumulative distribution for these events, $f_{i}^{\text {obs }}$, is just a sequence of rational fractions,

\footnotetext{
4 ITT Visual Information Solutions, ITT Industries, Inc.
}

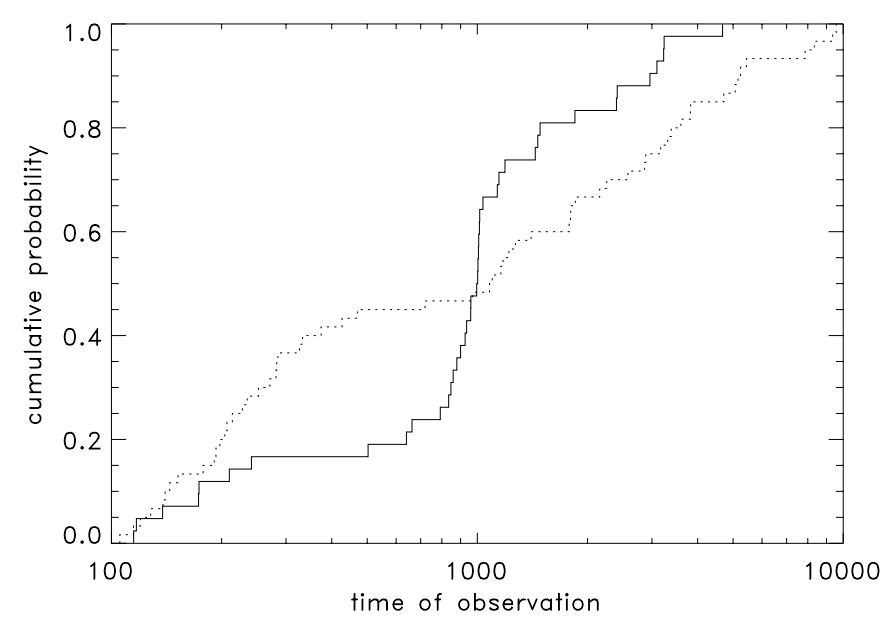

FIG. 2.-Cumulative distributions for the time of observation for detected afterglows (solid line) and undetected afterglows (dotted line) relative to the burst onset. The apparent step function for the detected events at $1000 \mathrm{~s}$ is an artifact of the selection criteria. Note that the undetected GRBs have a "best" limiting magnitude at a median time also close to $1000 \mathrm{~s}$. This is not a selection effect.

$\left(1,2,3, \ldots, n_{\text {det }}\right) / n_{\text {total }}$, where $n_{\text {det }}$ is the number of detected GRBs and $n_{\text {total }}$ is the number of all events considered, detected and undetected alike. The strategy to optimize the shape of $F(m)$ is now fairly simple: form the differences, $\delta_{i}=\left(f_{i}^{\text {calc }}-f_{i}^{\text {obs }}\right)$, and minimize the sum of squares, $\sum^{n_{\mathrm{det}}} \delta_{i}^{2}$. This last quantity defines the least-squares goodness-of-fit function that drives the downhill simplex routine mentioned previously. The montonicity of the cumulative distribution function helps ensure the stability of the optimum fit.

\section{RESULTS AND DISCUSSION}

The calculation described above was carried out with 4-7 degrees of freedom for the cubic spline representation, corresponding to dividing the range of $F,[0,1]$, into one to four equal segments. The resulting fit is shown in Figure 5 along with the actual GRB detections. The fits are qualitatively excellent.

The corresponding integral distribution function for the apparent optical brightness is shown in Figure 6. The curves all follow the same shape. The range of validity of these curves extends at least to the 90th percentile of the $m_{\text {det }}, 20.5$. At this point, the cumulative intrinsic afterglow distribution accounts for $57 \%$ of all Swift-identified GRBs. The most extreme useful point corresponds to the deepest detection at $m_{\text {det }}=22.1$, where the intrinsic distribution reaches $71 \%$. The remaining $29 \%$ may constitute two populations: GRBs inside optically dense regions or at redshifts beyond the Ly $\alpha$ cutoff. Since our statistical method relies on actual detections, the $29 \%$ could easily be somewhat lower and details of the high-magnitude afterglow distribution cannot be resolved. Similar conclusions about the population of dim or dark GRBs have been reached by others from far different arguments (Jakobsson et al. 2004, 2006). Thus, the original question of why half or less of all GRBs are optically identified has been resolved by the realization that roughly $25 \%$ are lost because they are dimmer than $m_{\mathrm{det}} \simeq 22$, and the rest are missed because the available instrumentation is inadequate. This partially answers one of the issues that led us to this analysis, our observations of the afterglow of GRB 060116 (Swan et al. 2006). Within $2000 \mathrm{~s}$, the afterglow became dimmer than $m_{R} \sim 22$, making it an exceedingly difficult target for further measurements. It is apparent that some but not all of the missing optical counterparts are due to such dim but detectable objects. 
TABLE 2

GRB Afterglow Nondetections

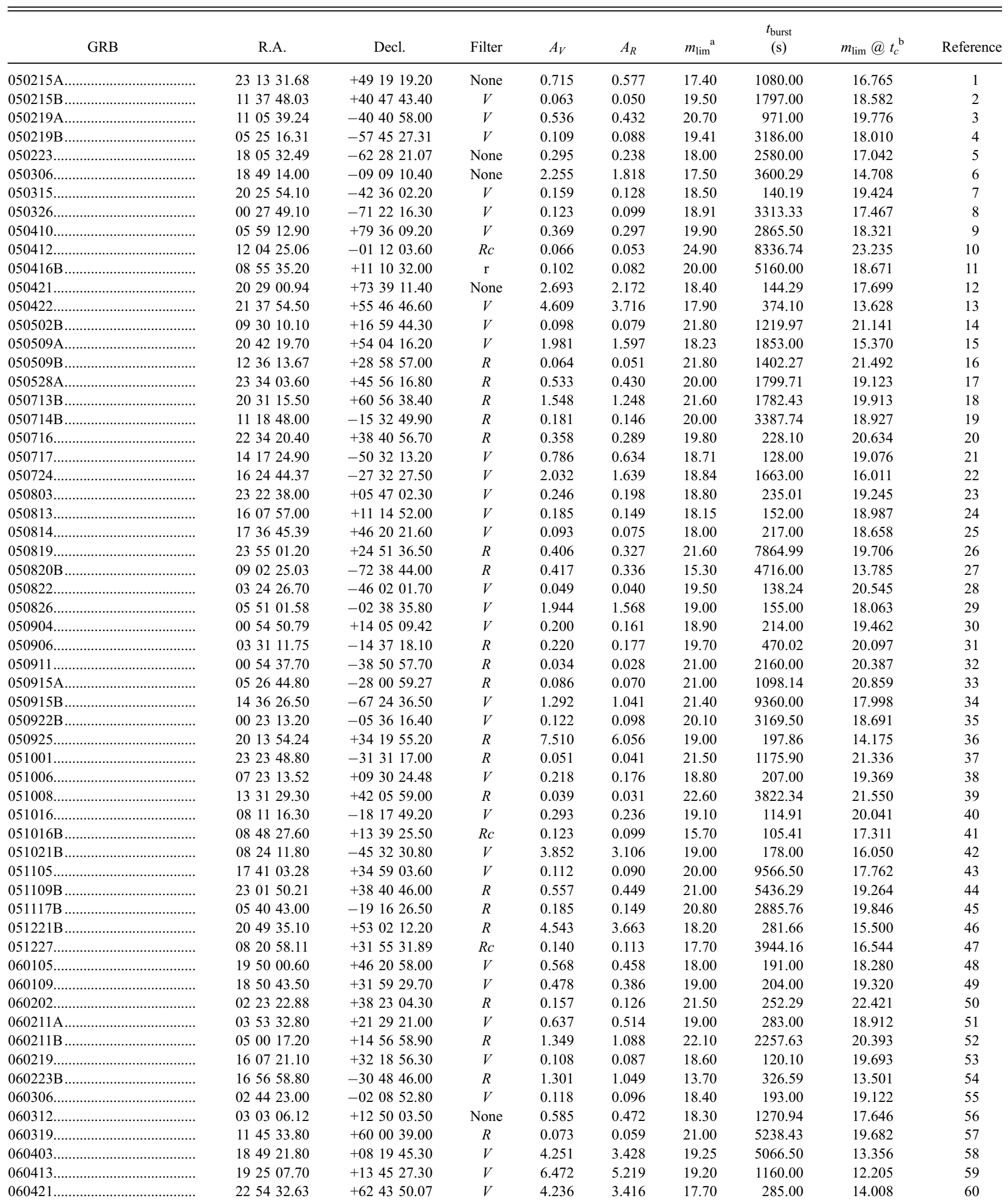


TABLE 2-Continued

\begin{tabular}{|c|c|c|c|c|c|c|c|c|c|}
\hline GRB & R.A. & Decl. & Filter & $A_{V}$ & $A_{R}$ & $m_{\lim }{ }^{\mathrm{a}}$ & $\begin{array}{c}t_{\text {burst }} \\
\text { (s) }\end{array}$ & $m_{\lim } @ t_{c}^{\mathrm{b}}$ & Reference \\
\hline $060427 \ldots \ldots$ & 081704.40 & +624018.30 & $V$ & 0.165 & 0.133 & 18.50 & 333.00 & 18.761 & 61 \\
\hline $060501 \ldots \ldots$ & 215329.90 & +435953.40 & None & 0.951 & 0.767 & 17.40 & 426.82 & 17.280 & 63 \\
\hline $060502 \mathrm{~B}$ & 183545.89 & +523756.20 & None & 0.145 & 0.117 & 20.00 & 719.71 & 20.133 & 64 \\
\hline 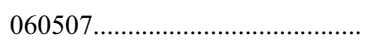 & 055951.70 & +751456.60 & $R$ & 0.514 & 0.414 & 19.20 & 3828.38 & 17.766 & 65 \\
\hline
\end{tabular}

NoтE.-Units of right ascension are hours, minutes, and seconds, and units of declination are degrees, arcminutes, and arcseconds.

a The quantity $m_{\text {lim }}$ is the magnitude upper limit at $t_{\text {burst }}$ seconds after the GRB trigger.

b The quantity $m_{\lim } @ t_{c}$ is the inferred value for $m_{\lim }$ at $t_{c}=1000 \mathrm{~s}$ after correcting for galactic absorption and average GRB color differences.

REFERENCES.- (1) Smith et al. 2005; (2) Roming et al. 2005; (3) Schady et al. 2005c; (4) Poole et al. 2005c; (5) Smith 2005; (6) Klotz et al. 2005c; (7) Rosen et al. 2005; (8) Holland et al. 2005b; (9) Boyd et al. 2005b; (10) Kosugi et al. 2005; (11) Berger et al. 2005; (12) Rykoff et al. 2005b; (13) McGowan et al. 2005b; (14) Cenko et al. 2005; (15) Poole et al. 2005a; (16) Wozniak et al. 2005; (17) Monfardini et al. 2005; (18) Lin et al. 2005; (19) Malesani et al. 2005; (20) Guidorzi et al. 2005a; (21) Hurkett et al. 2005; (22) Chester et al. 2005; (23) Brown et al. 2005a; (24) Retter et al. 2005a; (25) Retter et al. 2005b; (26) Bikmaev et al. 2005; (27) Jelinek et al. 2005; (28) Page et al. 2005; (29) Mangano et al. 2005; (30) Cucchiara et al. 2005; (31) Fox et al. 2005; (32) Tristram et al. 2005a; (33) Cenko \& Fox 2005; (34) Cobb \& Bailyn 2005; (35) de Pasquale et al. 2005; (36) Guidorzi et al. 2005c; (37) Tristram et al. 2005b; (38) Norris et al. 2005; (39) Rumyantsev et al. 2005a; (40) Boyd et al. 2005a; (41) Torii 2005b; (42) Retter et al. 2005c; (43) Brown et al. 2005b; (44) Huang et al. 2005; (45) Chen et al. 2005; (46) Klotz et al. 2005b; (47) Yanagisawa et al. 2005; (48) Ziaeepour et al. 2006; (49) de Pasquale et al. 2006b; (50) Monfardini et al. 2006b; (51) Hurkett et al. 2006; (52) Sharapov et al. 2006; (53) Breeveld \& Moretti 2006; (54) Torii \& Cummings 2006; (55) Angelini et al. 2006; (56) Schaefer et al. 2006; (57) Guziy et al. 2006; (58) Poole \& Boyd 2006; (59) Boyd et al. 2006; (60) Goad et al. 2006; (61) Mangano et al. 2006b; (62) Mangano et al. 2006a; (63) Rykoff et al. 2006a; (64) Zhai et al. 2006; (65) Halpern \& Mirabal 2006.

While recognizing that differentiation amplifies errors, it is still useful to look at the differential GRB afterglow magnitude distribution determined directly from the cumulative distribution discussed above. As shown in Figure 7, a peak appears at $m_{\mathrm{det}} \approx$ 19.5 , which is only slightly displaced from the peak in the actual observed $m_{\text {det }}$ distribution. One might argue that statistical errors in evaluating $m_{\lim }$ could shift this somewhat rightward, but unitarity puts limits on how much further the integral distribution can rise without changing slope. Thus, the overall behavior of the apparent GRB afterglow distribution is likely to follow closely the curves shown. Some caution should be exercised about overinterpreting the physical significance of this peak. Since the Burst Alert Telescope (BAT) detector on Swift operates in flux-limited mode, cutoffs at low brightness may simply be a reflection of a proportional correlation to lower fluxes in $\gamma$-rays.

We have described a statistical analysis of GRB optical afterglows that has attempted to obtain the brightness distribution

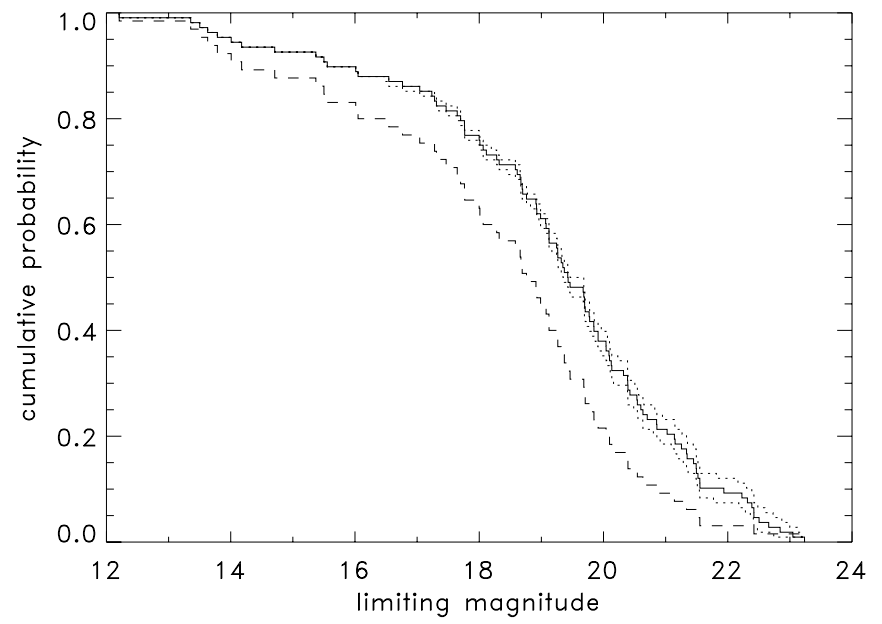

FIG. 3.-Cumulative distribution for observation limiting magnitudes. The dashed line corresponds to the distribution for the best sensitivity for each nondetection; the solid line is the estimate obtained for all observations, both detections and nondetections, using the iterative technique described in the text. The dotted lines show the first and third quartile distributions obtained in the Monte Carlo process. These clearly bracket the median quite closely. for observers on Earth to better understand the population of dimmer events and the criteria for improving such investigations. By including the distortion effects of instrumental characteristics and by comparing at a time accessible to almost all observers, our results are largely biased only by the trigger threshold of the BAT detector on board Swift. A rather different approach has been attempted by two groups during the past 2 years (Gendre \& Boër 2005; Nardini et al. 2006a, 2006b). Their aim is to find discriminants that would identify subclasses of GRB events by translating observed fluxes to the rest frame of the GRB. In particular, Nardini et al. have found that by using those events with redshift information, they could project the optical flux in $R$ band back to the GRB rest frame at a proper time of $12 \mathrm{hr}$. For a typical burst with $z \sim 2$, this corresponds to an observation 1.5 days following the burst trigger, $\sim 100$ times greater than the value of $t_{c}$ of $1000 \mathrm{~s}$ employed in our analysis. At this late epoch, they find that the majority of events are clustered in luminosity with a standard deviation of $0.70 \mathrm{mag}$. A low-luminosity population is also identified as a minority constituent of an apparently bimodal distribution

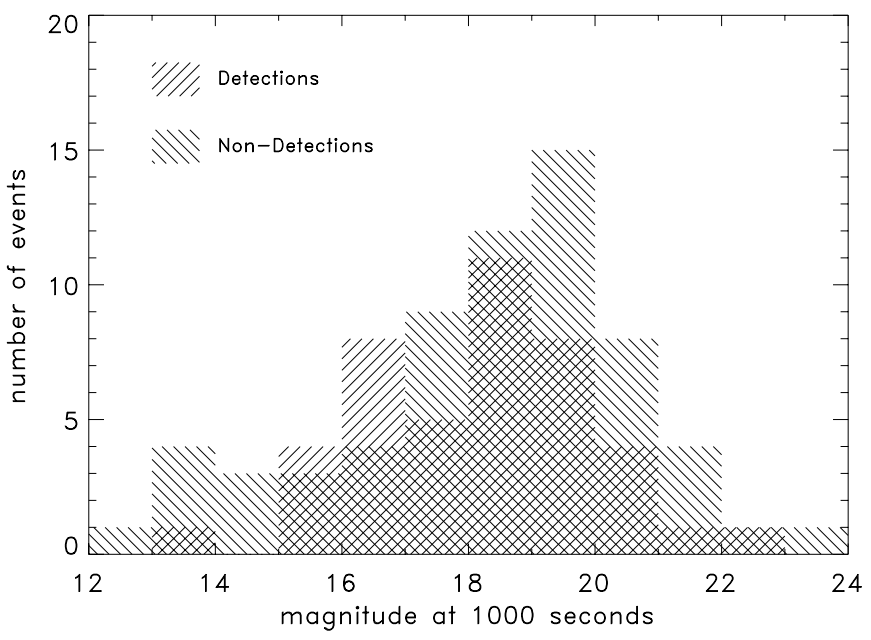

FIG. 4.-Histogram of GRB optical afterglow detections and nondetections transformed to $t_{c}=1000 \mathrm{~s}$. Despite a large overlap region, a substantial number of nondetections occur at limiting magnitudes deeper than most detections. 


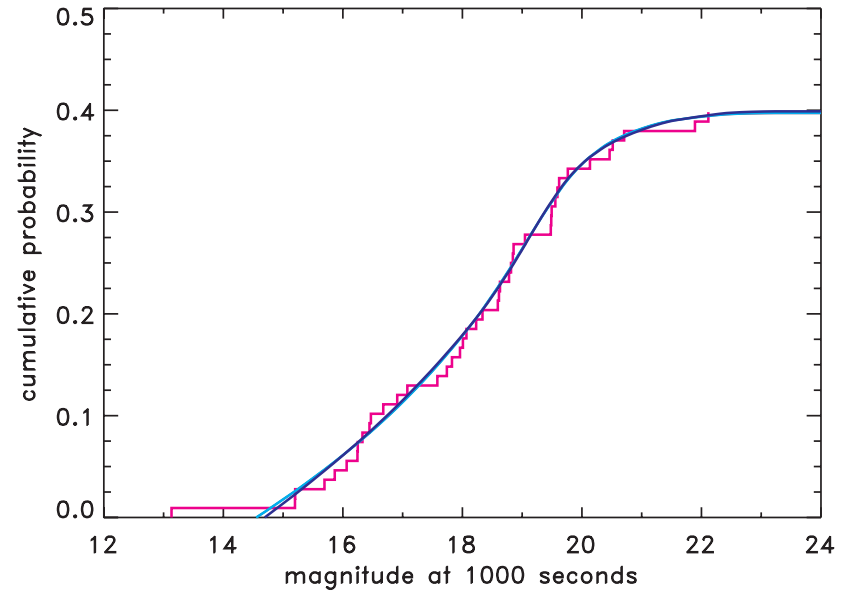

FIG. 5.-Cumulative distribution of detected GRB optical afterglows. The violet "staircase" line shows the experimentally observed distribution. The leastsquares estimates are shown in red, green, cyan, and blue, corresponding respectively to $4,5,6$, and 7 degrees of freedom of the B-spline representation.

and exhibits a factor of 15 lower flux. In their most recent paper, they include 25 Swift bursts, of which 17 are referenced in this present paper. The high-flux fraction of Nardini events has a mean observer frame brightness about $1 \mathrm{mag}$ greater than our entire detection sample, while the low-flux cluster, with only four events, is statistically indistinguishable. Given the different methods and goals of the Nardini analysis, no further comparison is likely to be meaningful.

\section{IMPLICATIONS FOR FUTURE GRB OBSERVATIONS}

Observations of GRBs are difficult and expensive primarily because of the reliance on large X-ray and $\gamma$-ray detectors in space such as Swift and GLAST, each of which costs a good fraction of a billion dollars. Recent history has shown that multiwavelength observations considerably enhance the amount of information about these elusive events. At the present time, we still do not have a definite theory of the energy transport within a GRB jetit could be baryonic, $e^{ \pm}$pairs, or electromagnetic Poynting flux.

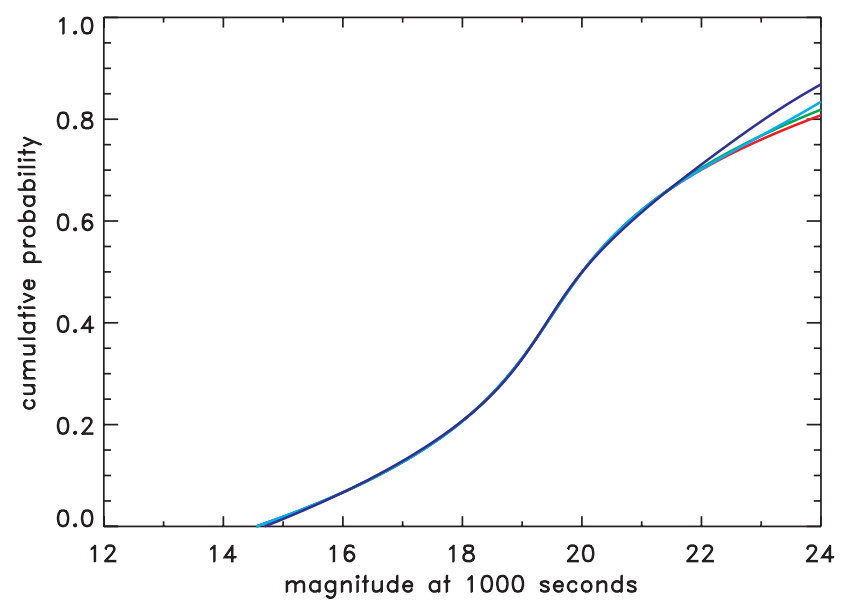

FIG. 6.- Intrinsic cumulative GRB apparent optical afterglow distribution. The colors, red, green, cyan, and blue, correspond respectively to B-spline curves with $4,5,6$, and 7 degrees of freedom. Crudely speaking, $71 \%$ of all GRB afterglows have $m_{R}<22.1$ at $t_{\mathrm{obs}}=1000 \mathrm{~s}$, the dimmest GRB optically detected.

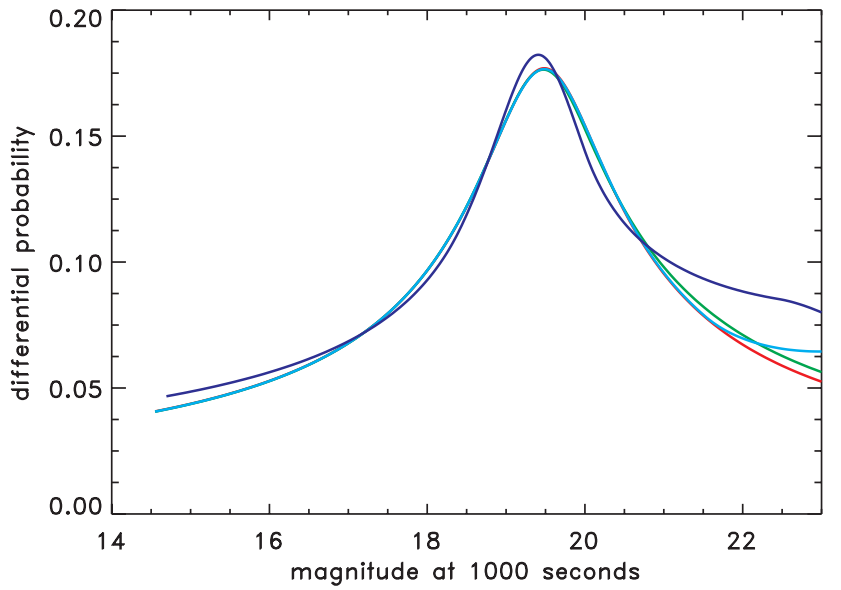

FIG. 7.-Differential GRB apparent optical afterglow distribution. The colors, red, green, cyan, and blue, correspond respectively to B-spline curves with 4, 5, 6, and 7 degrees of freedom. The peak at $m_{\text {det }} \approx 19.5$ seems to be an unavoidable feature.

Many hope that if GRBs are better understood, they could help improve our understanding of the early star formation period of our universe. In any case, research is bound to continue in this area for many years to come, although launching of new space missions dedicated to GRBs will likely be infrequent. The analysis in this paper suggests that a natural threshold sensitivity for optical observations of Swift-detected bursts is $m_{R} \approx 20$. The data gathered for this paper show that such levels are routinely achieved by $2 \mathrm{~m}$ telescopes. The cost of such instruments is in the neighborhood of $\$ 5$ million, especially if purchased in multiple units. The total number of such units can be gauged by the following simple argument: the sky is dark above any specific site for about $1 / 3$ of the day, a randomly detected GRB will be at an immediately accessible zenith angle about $1 / 3$ of the time, and the weather at a good site will be suitable with probability of $3 / 5$. The joint probability of all three independent conditions is $1 / 15$, implying that optimal coverage is achieved with $\approx 15$ instruments globally distributed around the Earth. The overall optical detection probability is modified to some extent by details of $\gamma$-ray detector pointing constraints. Clearly, a number of areas on Earth are already well populated with research-grade telescopes, particularly Chile and southwestern United States. Many parts of the world are not so well blessed. Some nations such as Thailand and Iran have recognized the scientific niche for observing optical transients and expect to install $2 \mathrm{~m}$ optical telescopes within a few years. That still leaves a number of sites in Asia and elsewhere that could successfully enhance global coverage of rare phenomena such as GRBs. An alternative is to launch rapid-response optical/IR telescopes in space that would obviate the need for ground-based facilities. Unfortunately, the cost of even a modest $1 / 2 \mathrm{~m}$ aperture telescope far exceeds installing two dozen much larger instruments located on Earth.

Any instrument dedicated to GRB optical afterglow detection must be robotic, with a slew time of tens of seconds in order to maximize the time overlap with the most variable periods of $\mathrm{X}$-ray and $\gamma$-ray emission. Such a telescope would be more useful for a broader range of research if the field of view (FOV) can be kept large, at least a square degree. The best example for this argument is the Sloan Digital Sky Survey, whose telescope primary has an aperture of $2.5 \mathrm{~m}$ and a FOV of $1.5 \mathrm{deg}^{2}$. To complete this picture, the imaging focal plane could be populated with a $2 \times$ 2 array of large format silicon CCDs. This would be even more 
useful if the instrument could operate as a two-band system with a dichroic splitter to separate $R$ band and $I$ or $J$ band to two different cameras. Such multiband coverage might better elucidate the origin of "dark" bursts, whether hidden by optical extinction of dense molecular clouds or redshifted and destroyed by Ly $\alpha$ absorption edges. Instruments such as described above run counter to the current government funding trend to shut down many $2 \mathrm{~m}$ telescopes in favor of fewer but more powerful $8 \mathrm{~m}$ class and larger. Such policies work well for the majority of astronomical objects that evolve exceedingly slowly with time but are inappropriate for relatively rare events with durations of minutes or seconds. It also behooves agencies such as NASA that fund space missions to help organize ground-based programs that will optimize the entire scientific return on investment.

The authors gratefully acknowledge the extensive help of Fang Yuan, Sarah Yost, Wiphu Rujopakarn, and Eli Rykoff in compiling the lists of optical observations used for this analysis as well as their comments and suggestions. This research was supported by NSF/AFOSR grant AST 03-35588, NASA grant NNG-04WC41G, NSF grant AST 04-07061, and the Michigan Space Grant Consortium.

\section{REFERENCES}

Angelini, L., et al. 2006, GCN Circ. 4848, http://gcn.gsfc.nasa.gov/gcn/gcn3/ 4848.gen3

Antonelli, L. A., et al. 2006, A\&A, 456, 509

Berger, E., Mulchaey, J., Morrell, N., \& Krzeminski, W. 2005, GCN Circ. 3283, http://gcn.gsfc.nasa.gov/gcn/gcn $3 / 3283$.gcn3

Bersier, D., et al. 2003, ApJ, 584, L43

Bikmaev, I., et al. 2005, GCN Circ. 3831, http:/gcn.gsfc.nasa.gov/gcn/gcn3/ 3831.gen3

. 2006, GCN Circ. 4652, http://gcn.gsfc.nasa.gov/gcn/gcn3/4652.gcn3

Boyd, P., Weigand, R. E., Holland, S. T., \& Blustin, A. 2006, GCN Circ. 4958, http://gcn.gsfc.nasa.gov/gcn/gcn3/4958.gcn3

Boyd, P., et al. 2005a, GCN Circ. 4096, http://gcn.gsfc.nasa.gov/gen/gcn3/4096 .gen 3

— 2005b, GCN Circ. 3230, http://gcn.gsfc.nasa.gov/gcn/gcn3/3230.gcn3 Breeveld, A., \& Moretti, A. 2006, GCN Circ. 4798, http://gcn.gsfc.nasa.gov/gcn/ gcn3/4798.gcn3

Brown, P. J., Band, D. L., Boyd, P., Norris, J., Hurley, K., \& Gehrels, N. 2005a, GCN Circ. 3759, http://gcn.gsfc.nasa.gov/gcn/gcn3/3759.gcn3

Brown, P. J., Mineo, T., Chester, M., Angelini, L., Meszaros, P., \& Gehrels, N. 2005b, GCN Circ. 4200, http://gcn.gsfc.nasa.gov/gcn/gcn3/4200.gcn3

Burenin, R., et al. 2003, GCN Circ. 2001, http:/gcn.gsfc.nasa.gov/gen/gcn3/ 2001.gen3

Cenko, S. B., \& Fox, D. B. 2005, GCN Circ. 3981, http://gcn.gsfc.nasa.gov/gen/ gcn3/3981.gcn 3

Cenko, S. B., Fox, D. B., Rich, J., Schmidt, B., Christiansen, J., \& Berger, E. 2005, GCN Circ. 3357, http://gcn.gsfc.nasa.gov/gen/gen3/3357.gcn3

Cenko, S. B., Ofek, E. O., \& Fox, D. B. 2006a, GCN Circ. 5048, http://gen.gsfe .nasa.gov/gen $/ \mathrm{gcn} 3 / 5048 . \mathrm{gcn} 3$

Cenko, S. B., et al. 2006b, ApJ, 652, 490

Chen, Y. T., Huang, K. Y., Ip, W. H., Urata, Y., Qiu, Y., \& Lou, Y. Q. 2005, GCN Circ. 4285, http://gcn.gsfc.nasa.gov/gen/gen3/4285.gen3

Chester, M., Covino, S., Schady, P., Roming, P., \& Gehrels, N. 2005, GCN Circ. 3670, http://gen.gsfc.nasa.gov/gen/gen3/3670.gen3

Cobb, B. E. 2006, GCN Circ. 4872, http://gen.gsfc.nasa.gov/gen/gen3/4872.gcn3

Cobb, B. E., \& Bailyn, C. D. 2005, GCN Circ. 3994, http://gcn.gsfc.nasa.gov/ $\mathrm{gcn} / \mathrm{gcn} 3 / 3994 . \mathrm{gcn} 3$

Covino, S., et al. 2003, A\&A, 404, L5

Cucchiara, A., et al. 2005, GCN Circ. 3923, http:/gcn.gsfc.nasa.gov/gcn/gcn3/ 3923.gcn3

de Pasquale, M., Norris, J., Kennedy, T., Mason, K., \& Gehrels, N. 2005, GCN Circ. 4028, http://gcn.gsfc.nasa.gov/gen/gcn3/4028.gen3

de Pasquale, M., et al. 2006a, MNRAS, 370, 1859

2006b, GCN Circ. 4455, http://gcn.gsfc.nasa.gov/gcn/gcn3/4455.gcn3

Eadie, W. T., Dryard, D., James, F. E., Roos, M., \& Sadoulet, B. 1971, Statistical Methods in Experimental Physics (Amsterdam: North-Holland)

Fox, D. B., Cenko, S. B., \& Schmidt, B. P. 2005, GCN Circ. 3931, http://gcn.gsfe .nasa.gov/gen $/ g \mathrm{cn} 3 / 3931 . \mathrm{gcn} 3$

Fynbo, J. U., et al. 2001, A\&A, 369, 373

Gendre, B., \& Boër, M. 2005, A\&A, 430, 465

Goad, M. R., et al. 2006, GCN Circ. 4985, http:/gcn.gsfc.nasa.gov/gcn/gcn3/ 4985.gcn3

Guetta, D., et al. 2007, A\&A, 461, 95

Guidorzi, C., et al. 2005a, GCN Circ. 3625, http://gen.gsfc.nasa.gov/gen/gen3/ 3625.gcn 3

2005b, ApJ, 630, L121

2005c, GCN Circ. 4035, http://gcn.gsfc.nasa.gov/gcn/gcn3/4035.gcn3

. 2006, GCN Circ. 4661, http://gen.gsfc.nasa.gov/gen/gen3/4661.gen3

Guziy, S., Gómez Velarde, G., \& Castro-Tirado, A. J. 2006, GCN Circ. 4896, http://gcn.gsfc.nasa.gov/gen/gen3/4896.gen3

Halpern, J. P., \& Mirabal, N. 2006, GCN Circ. 5086, http:/gcn.gsfc.nasa.gov/ $\mathrm{gcn} / \mathrm{gcn} 3 / 5086 . \mathrm{gcn} 3$
Holland, S. T., Band, D., Mason, K., Marshall, F., \& Gehrels, N. 2005a, GCN Circ. 4300, http://gcn.gsfc.nasa.gov/gen/gen3/4300.gen3

Holland, S. T., et al. 2005b, GCN Circ. 3150, http://gcn.gsfc.nasa.gov/gen/gen3/ 3150. $\mathrm{gcn} 3$

Huang, F. Y., Huang, K. Y., Ip, W. H., Urata, Y., Qiu, Y., \& Lou, Y. Q. 2005, GCN Circ. 4231, http://gcn.gsfc.nasa.gov/gcn/gcn3/4231.gcn3

Hurkett, C., et al. 2005, GCN Circ. 3633, http:/gen.gsfc.nasa.gov/gen/gen3/ 3633.gcn 3

. 2006, GCN Circ. 4736, http://gcn.gsfc.nasa.gov/gen/gcn3/4736.gcn3

Jakobsson, P., Hjorth, J., Fynbo, J. P. U., Watson, D., Pedersen, K., Björnsson, G., \& Gorosabel, J. 2004, ApJ, 617, L21

Jakobsson, P., et al. 2006, A\&A, 447, 897

Jelinek, M., Prouza, M., Kubanek, P., Nekola, M., \& Hudec, R. 2005, GCN Circ. 3854, http://gcn.gsfc.nasa.gov/gen/gcn3/3854.gcn3

Jelínek, M., et al. 2006, A\&A, 454, L119

Kendall, M., \& Stuart, A. 1979, The Advanced Theory of Statistics, Vol. 2: Inference and Relationship (4th ed.; London: Griffin)

Klose, S., et al. 2003, ApJ, 592, 1025

Klotz, A., Boer, M., \& Atteia, J. L. 2005a, GCN Circ. 3403, http://gcn.gsfc.nasa.gov/ $\mathrm{gcn} / \mathrm{gcn} 3 / 3403$.gcn3

- 2005b, GCN Circ. 4386, http://gcn.gsfc.nasa.gov/gcn/gcn3/4386.gcn3 2006a, GCN Circ. 4483, http://gen.gsfc.nasa.gov/gen/gen3/4483.gen3

Klotz, A., Boer, M., Atteia, J. L., \& Stratta, G. 2005c, GCN Circ. 3084, http://gen .gsfc.nasa.gov/gen $/ \mathrm{gcn} 3 / 3084 . \mathrm{gcn} 3$

Klotz, A., Boër, M., Atteia, J. L., Stratta, G., Behrend, R., Malacrino, F., \& Damerdji, Y. 2005d, A\&A, 439, L35

Klotz, A., Gendre, B., Stratta, G., Atteia, J. L., Boër, M., Malacrino, F., Damerdji, Y., \& Behrend, R. 2006b, A\&A, 451, L39

Koppelman, M. 2006, GCN Circ. 4977, http://gcn.gsfc.nasa.gov/gcn/gen3/4977 . $\mathrm{gcn} 3$

Kosugi, G., Kawai, N., Aoki, K., Hattori, T., Ohta, K., \& Yamada, T. 2005, GCN Circ. 3263, http://gcn.gsfc.nasa.gov/gen/gen3/3263.gen3

Levan, A., et al. 2006, ApJ, 647, 471

Li, W. 2006, GCN Circ. 4499, http://gcn.gsfc.nasa.gov/gen/gen3/4499.gen3

Li, W., Chornock, R., Butler, N., Bloom, J., \& Filippenko, A. V. 2006, GCN Circ. 5027, http://gcn.gsfc.nasa.gov/gcn/gcn3/5027.gcn3

Lin, Z. Y., Huang, K. Y., Ip, W. H., Urata, Y., Qiu, Y., \& Lou, Y. Q. 2005, GCN Circ. 3593, http://gcn.gsfc.nasa.gov/gen/gen3/3593.gen3

Lipunov, V., et al. 2005, GCN Circ. 3883, http://gcn.gsfc.nasa.gov/gen/gen3/ 3883.gcn 3

Malesani, D., D’Avanzo, P., Israel, G. L., Piranomonte, S., Chincarini, G., Stella, L., Tagliaferri, G., \& Depagne, E. 2005, GCN Circ. 3614, http://gen.gsfc.nasa .gov/gen/gen $3 / 3614 . g c n 3$

Mangano, V., et al. 2005, GCN Circ. 3884, http://gcn.gsfc.nasa.gov/gen/gcn3/ 3884.gen 3

2006a, GCN Circ. 5014, http://gen.gsfc.nasa.gov/gen/gen3/5014.gen3 2006b, GCN Circ. 5006, http://gcn.gsfc.nasa.gov/gcn/gcn3/5006.gcn3

Marshall, F., Boyd, P., Chester, M., \& Cummings, J. 2006, GCN Circ. 4814, http://gcn.gsfc.nasa.gov/gen/gen3/4814.gen3

McGowan, K., Band, D., Brown, P., Gronwall, C., Huckle, H., \& Hancock, B. 2005a, GCN Circ. 3739, http://gcn.gsfc.nasa.gov/gen/gcn3/3739.gcn3

McGowan, K., et al. 2005b, GCN Circ. 3317, http://gcn.gsfc.nasa.gov/gcn/gen3/ 3317.gcn 3

Monfardini, A., et al. 2005, GCN Circ. 3503, http://gcn.gsfc.nasa.gov/gcn/gcn3/ 3503.gcn 3

2006a, ApJ, 648, 1125

2006b, GCN Circ. 4630, http://gen.gsfc.nasa.gov/gen/gen3/4630.gcn3

Nardini, M., Ghisellini, G., Ghirlanda, G., Tavecchio, F., Firmani, C., \& Lazzati, D. 2006a, A\&A, 451, 821

. 2006b, in Proc. SWIFT and GRBs: Unveiling the Relativistic Universe, submitted (astro-ph/0612486) 
Nelder, J. A., \& Mead, R. 1965, Comput. J., 7, 308

Norris, J., et al. 2005, GCN Circ. 4061, http://gen.gsfc.nasa.gov/gen/gen3/ 4061.gen 3

Oates, S. R., et al. 2006, MNRAS, 372, 327

Pagani, C., et al. 2006, ApJ, 645, 1315

Page, M. J., Ziaeepour, H., Blustin, A. J., Chester, M., Fink, R., \& Gehrels, N. 2005, GCN Circ. 3859, http://gcn.gsfc.nasa.gov/gen/gcn3/3859.gcn3

Pandey, S. B., et al. 2006, A\&A, 460, 415

Poole, T. S., \& Boyd, P. T. 2006, GCN Circ. 4951, http://gcn.gsfc.nasa.gov/gen/ gcn3/4951.gcn3

Poole, T., Hurkett, C., Hunsberger, S., Breeveld, A., Boyd, P., Gehrels, N., Mason, K., \& Nousek, J. 2005a, GCN Circ. 3394, http://gcn.gsfc.nasa.gov/ $\mathrm{gcn} / \mathrm{gcn} 3 / 3394 . \mathrm{gcn} 3$

Poole, T., Moretti, A., Holland, S. T., Chester, M., Angelini, L., \& Gehrels, N. 2005b, GCN Circ. 3698, http://gcn.gsfc.nasa.gov/gen/gcn3/3698.gcn3

Poole, T., et al. 2005c, GCN Circ. 3050, http://gcn.gsfc.nasa.gov/gen/gcn3/ 3050.gcn 3

Press, W. H., Teukolsky, S. A., Vetterling, W. T., \& Flannery, B. P. 1992, Numerical Recipes in C: The Art of Scientific Computing (2nd ed.; Cambridge: Cambridge Univ. Press)

Quimby, R., McMahon, E., \& Murphy, J. 2003, GCN Circ. 2298, http://gcn gsfc.nasa.gov/gen $/ g \mathrm{gn} 3 / 2298 . \mathrm{gcn} 3$

Quimby, R., Schaefer, B. E., \& Swan, H. 2006a, GCN Circ. 4782, http://gen gsfc.nasa.gov $/ \mathrm{gcn} / \mathrm{gcn} 3 / 4782 . \mathrm{gcn} 3$

Quimby, R. M., et al. 2006b, ApJ, 640, 402

Reichart, D. E., \& Price, P. A. 2002, ApJ, 565, 174

Retter, A., et al. 2005a, GCN Circ. 3788, http://gcn.gsfc.nasa.gov/gen/gen3/3788 .gcn3

2005b, GCN Circ. 3799, http://gcn.gsfc.nasa.gov/gcn/gcn3/3799.gen3 2005c, GCN Circ. 4126, http://gcn.gsfc.nasa.gov/gcn/gcn3/4126.gcn3

Rol, E., Wijers, R. A. M. J., Kouveliotou, C., Kaper, L., \& Kaneko, Y. 2005, ApJ, 624,868

Romano, P., et al. 2006, A\&A, 456, 917

Roming, P., et al. 2005, GCN Circ. 3026, http://gcn.gsfc.nasa.gov/gen/gcn3/ 3026.gen 3

Rosen, S., et al. 2005, GCN Circ. 3095, http://gen.gsfc.nasa.gov/gen/gen3/3095 .gcn 3

Rumyantsev, V., Biryukov, V., Pozanenko, A., \& Ibrahimov, M. 2005, GCN Circ. 4087, http://gcn.gsfc.nasa.gov/gen/gen3/4087.gen3

Rumyantsev, V., Pavlenko, E., Efimov, Y., Antoniuk, K., Antoniuk, O., Primak, N., \& Pozanenko, A. 2003, GCN Circ. 2005, http://gcn.gsfc.nasa.gov/gcn/ gen $3 / 2005 . g c n 3$

Rykoff, E. S., Schaefer, B. E., Yost, S. A., \& Quimby, R. 2006a, GCN Circ. 5041, http://gcn.gsfc.nasa.gov/gen/gen3/5041.gen3

Rykoff, E. S., Yost, S. A., Rujopakarn, W., Quimby, R., Smith, D. A., \& Yuan, F. 2005a, GCN Circ. 4012, http://gen.gsfc.nasa.gov/gen/gcn3/4012.gen3

Rykoff, E. S., Yost, S. A., \& Swan, H. 2005b, GCN Circ. 3304, http://gcn.gsfc .nasa.gov/gen $/ \mathrm{gcn} 3 / 3304 . \mathrm{gcn} 3$

Rykoff, E. S., et al. 2005c, ApJ, 631, L121 . 2006b, ApJ, 638, L5
Schady, P., Sakamoto, T., McGowan, K., Boyd, P., Roming, P., Nousek, J., \& Gehrels, N. 2005a, GCN Circ. 3276, http://gcn.gsfc.nasa.gov/gen/gcn3/3276.gcn3

Schady, P., et al. 2005b, GCN Circ. 3817, http://gcn.gsfc.nasa.gov/gen/gen3/ 3817.gcn 3

. 2005c, GCN Circ. 3039, http://gen.gsfc.nasa.gov/gen/gen3/3039.gen3 2006, ApJ, 643, 276

Schaefer, B. E., Yuan, F., Yost, S. A., \& Quimby, R. 2006, GCN Circ. 4860, http:// gen.gsfc.nasa.gov/gen/gen3/4860.gen3

Schlegel, D. J., Finkbeiner, D. P., \& Davis, M. 1998, ApJ, 500, 525

Sharapov, D., Ibrahimov, M., \& Pozanenko, A. 2006, GCN Circ. 4925, http://gcn .gsfc.nasa.gov/gcn/gen $3 / 4925$.gcn3

Smith, D. A. 2005, GCN Circ. 3056, http://gcn.gsfc.nasa.gov/gcn/gcn3/3056 gcn3

Smith, D. A., Rykoff, E. S., \& Yost, S. A. 2005, GCN Circ. 3021, http://gen .gsfc.nasa.gov/gcn $/ g \mathrm{cn} 3 / 3021 . \mathrm{gcn} 3$

Stanek, K. Z., Garnavich, P. M., Kaluzny, J., Pych, W., \& Thompson, I. 1999, ApJ, 522, L39

Stanek, K. Z., et al. 2007, ApJ, 654, L21

Still, M., et al. 2005, ApJ, 635, 1187

Swan, H., Akerlof, C., Rykoff, E., Yost, S., \& Smith, I. 2006, GCN Circ. 4568, http://gcn.gsfc.nasa.gov/gcn/gen3/4568.gcn3

Torii, K. 2005a, GCN Circ. 3943, http://gcn.gsfc.nasa.gov/gen/gen3/3943.gen3 . 2005b, GCN Circ. 4112, http://gcn.gsfc.nasa.gov/gen/gen3/4112.gcn3

Torii, K., \& Cummings, J. 2006, GCN Circ. 4826, http://gcn.gsfc.nasa.gov/gcn/ gcn3/4826.gen3

Tristram, P., Castro-Tirado, A. J., Guziy, S., de Ugarte Postigo, A., Jelinek, M., Gorosabel, J., \& Yock, P. 2005a, GCN Circ. 3965, http://gcn.gsfc.nasa.gov/ $\mathrm{gcn} / \mathrm{gcn} 3 / 3965 . \mathrm{gcn} 3$

Tristram, P., Jelinek, M., Castro-Tirado, A. J., de Ugarte Postigo, A., Guziy, S., Gorosabel, J., Yock, P., \& Krimm, H. 2005b, GCN Circ. 4055, http://gcn.gsfc .nasa.gov/gen $/ \mathrm{gcn} 3 / 4055 . \mathrm{gcn} 3$

van Paradijs, J., et al. 1997, Nature, 386, 686

Vergani, S. D., Molinari, E., Zerbi, F. M., \& Chincarini, G. 2004, A\&A, 415, 171

Wozniak, P., Vestrand, W. T., Wren, J., Evans, S., \& White, R. 2005, GCN Circ. 3414, http://gen.gsfc.nasa.gov/gen/gen3/3414.gen3

Wren, J., Vestrand, W. T., White, R., Wozniak, P., \& Evans, S. 2005, GCN Circ. 4380, http://gen.gsfc.nasa.gov/gen/gen3/4380.gen3

Yanagisawa, K., Sakamoto, T., \& Kawai, N. 2005, GCN Circ. 4418, http://gen .gsfc.nasa.gov/gcn/gcn3/4418.gcn3

Yanagisawa, K., Toda, H., \& Kawai, N. 2006, GCN Circ. 4517, http://gen.gsfc nasa.gov/gen $/ \mathrm{gcn} 3 / 4517 . \mathrm{gcn} 3$

Yost, S. A., et al. 2007, ApJ, 657, 925

Zhai, M., Qiu, Y. L., Wei, J. Y., Hu, J. Y., Deng, J. S., Wang, X. F., Huang, K. Y., \& Urata, Y. 2006, GCN Circ. 5057, http://gcn.gsfc.nasa.gov/gcn/gcn3/5057.gcn3

Zharikov, S., Benitez, E., Torrealba, J., \& Stepanian, J. 2003, GCN Circ. 2022, http://gcn.gsfc.nasa.gov/gen/gcn3/2022.gcn3

Zheng, W. K., Zhai, M., Qiu, Y. L., Wei, J. Y., Hu, J. Y., \& Deng, J. S. 2006, GCN Circ. 4930, http://gcn.gsfc.nasa.gov/gen/gcn3/4930.gcn3

Ziaeepour, H., et al. 2006, GCN Circ. 4429, http://gcn.gsfc.nasa.gov/gcn/gcn3/ 4429.gen3 